\title{
Direct energy transfer from excited organic adsorbates to intrinsic defect sites in silicalite
}

\author{
A. R. Leheny and N. J. Turro \\ Department of Chemistry, Columbia University, New York, New York 10027 \\ J. M. Drake \\ Exxon Research and Engineering Company, Clinton Township, Annandale, New Jersey 08801
}

(Received 22 January 1992; accepted 12 May 1992)

\begin{abstract}
The decay of the excited singlet state of an aromatic molecule such as naphthalene adsorbed to the surface of silica is nonexponential. This nonexponential decay is shown to result from a multipolar interaction between the excited adsorbate and the intrinsic defects of silica. We present experimental evidence for direct energy transfer between the donor molecules, naphthalene and 2-methoxynaphthalene, and acceptor defects sites of a crystalline silica zeloite (silicalite). The principal condition for direct energy transfer, spectral overlap between the optical absorption of the defects and the fluorescence emission of the adsorbate is demonstrated for this system, and the nonexponential relaxation of the donor is shown to be described by a Forster-type stretched exponential. Based on the spectral overlap and energy transfer rate determined, the intrinsic defect density of silicalite is calculated to be $3 \pm 2 \times 10^{20} \mathrm{~cm}^{-3}$.
\end{abstract}

\section{INTRODUCTION}

The dynamic properties of molecular probes in microporous and microheterogeneous systems have received a great deal of experimental ${ }^{1-12}$ and theoretical ${ }^{13-18}$ attention. Systems studied have included zeolites, silica gels, micelles, polymers, and biopolymers, porous glass and mixed crystals. ${ }^{1-12}$ Models have been developed to treat the role of spatial restrictions on the dynamics of these systems and to characterize the morphology of these structures. ${ }^{13,14}$ In addition to spatial restrictions, the interactions between the probes and the substrate have been found to contribute to the complexity of the observed behavior. ${ }^{10-12}$

In this paper, we present experimental evidence which suggests that under the appropriate conditions, the excited state of a molecule adsorbed to a silica zeolite (silicalite) interacts with the intrinsic defect sites of the silica leading to a nonexponential survival probability $\Phi(t)$. The interaction is proposed to be an energy transfer quenching reaction

$$
D^{*}+A \stackrel{W^{\prime}(r)}{\rightarrow} A^{*}+D,
$$

where the adsorbed aromatic molecules are the donors $\left(D^{*}, D\right)$ and the defect sites are the acceptors $\left(A, A^{*}\right)$. $W(r)$ describes the hierarchical one step energy transfer rate which depends on the distance $r$ between $D^{*}$ and $A$.

It is not obvious a priori which mechanism, a multipolar or an exchange interaction, dominates the energy transfer process in our system. We show that when the basic requirement for either energy transfer mechanism is fulfilled there is a spectral overlap between the fluorescence emission of the adsorbed donor molecule and a subbandgap absorption of the intrinsic silica defects specifically dangling peroxide radicals $\left(\mathrm{SiO}_{2} \cdot{ }^{\circ}\right)$ and peroxide bridges ( $\mathrm{Si}-\mathrm{O}-\mathrm{O}-\mathrm{Si}$ ). To determine which energy transfer process gives rise to the nonexponential decay observed, we fit the measured survival probability data for each donor to model decay laws for each mechanism. Because dipolar and exchange energy transfer depend in unique ways on the separation between $D^{*}$ and $A, W(r)$ gives decay laws that are distinctly different.

The formal derivation of these decay laws can be found in the literature and we present a brief description of how each energy transfer process leads to different form of the donors survival probability. The general form of the survival probability for a donor in the presence of acceptors is $^{1,13,14}$

$$
\begin{aligned}
\Phi_{D}\left(t, r_{0}\right)= & \exp \left(-p \int d r \rho_{0}(r)\right. \\
& \left.\times\left\{1-\exp \left[-W\left(r-r_{0}\right) t\right]\right\}\right),
\end{aligned}
$$

where we exclude the position of the donor $r_{0}$, and $p$ is the fraction of the total number of acceptor sites that are occupied. We consider the case where the energy transfer [Eq. (1)] is a one-step process with no significant back transfer and $p<1$. For donor-acceptor interaction in restricted geometries such as porous silicas, it is necessary to identify the form of the site density function $\rho_{0}(r)$ which describes the spatial agrrangement of the acceptors around the donor. $\rho_{0}(r)$ is essentially the two-point correlation function on the confining structure. Therefore, for each geometry, one must evaluate the integral appearing in $\mathrm{Eq}$. (2)

$$
J\left(t, r_{0}\right)=\int d r \rho_{0}(r)\left\{1-\exp \left[-W\left(r-r_{0}\right) t\right\} .\right.
$$

In the case of silicalite as the host geometry, we will make a number of assumptions about the approximate form of $\rho_{0}(r)$ which do not compromise our general conclusions. 
We now must consider the form of $W(r)$ for each of the energy transfer mechanisms. For multipolar interactions,

$$
W(r)=\frac{3}{2} \kappa^{2} \frac{1^{4}}{\tau_{0}}\left(\frac{R_{0}}{r}\right)^{S},
$$

where for dipole-dipole interactions $S=6, \tau_{0}$ is the isolated donor lifetime, $k$ is the anisotropic factor containing the angular dependence of the dipolar interaction, and $R_{0}$ is the critical transfer radius or Forster radius. $R_{0}$ is defined as $^{1,13-16}$

$$
R_{0}^{6}=\frac{9000 \ln 10 \kappa^{2} Q_{D}}{128 \pi^{6} n^{4} N_{A}} \int \frac{F_{D}(v) \epsilon_{A}(v)}{v^{4}} d v,
$$

where $N_{A}$ is Avagadro's number, $n$ is the refractive index of the medium, $F_{D}(v)$ is the fluorescence intensity of the donor in wave numbers normalized as $\int F_{D}(v) d v=1 . Q_{D}$ is the quantum yield of the donor fluorescence and $\epsilon_{A}(v)$ is the acceptor molar extinction coefficient. In the limit of an infinite system with a random acceptor distribution $r_{0}(r)$ $=r_{0}$, we recover a general form of the survival probability for multipolar interactions in a three-dimensional system. ${ }^{1.13-16}$

$$
\Phi(t)=\exp \left\{-\frac{t}{\tau_{0}}-\left[A_{0} \Gamma(1-d / S)\left(\frac{t}{\tau_{0}}\right)^{d / S}\right]\right\},
$$

where $d$ is the dimension of the embedding medium, $\Gamma$ is the gamma function, and the prefactor $A_{0}$ is equal to ${ }^{1,13-16}$

$$
A_{0}=p \rho_{0} C \pi R_{0}^{d}
$$

which defines the number of acceptor defect sites in a sphere of radius $R_{0}$ with $C$ a constant that depends on the dimension $d$ (e.g., for $d=3, C=4 / 3$ ). From Eq. (5), it is clear that when the interactions between the excited aromatic molecules and the defects of silica are dipolar, the normal exponential decay is modified at early times. The decay curves under the assumption of a dipolar interaction have the characteristic form of a stretched exponential with exponent $\alpha$ damped by an exponential ${ }^{1,13-17}$

$$
\Phi(t)=\exp \left(\frac{-t}{\tau_{0}}\right) \exp \left[-A_{0}\left(\frac{t}{\tau_{0}}\right)^{\alpha}\right]
$$

This stretched exponential nature of $\Phi(t)$ originates from the hierarchy of donor-acceptor distances and from the scaling behavior of $W(r)$ [see Eq. (4)].

When the donor-acceptor interactions are exchange in origin, the temporal nature of the donor survival probability is not stretched exponential in character. For exchange interactions, the transfer rate $W(r)$ between donors and acceptors depends exponentially on their separation distance

$$
W(r)=\frac{A}{\tau_{0}} \exp (-\epsilon r),
$$

where $\epsilon$ is a measure of the range of the exchange interaction. Substituting the form of $W(r)$ given by Eq. (9) into Eq. (2) as done in Refs. 18 and 19 yields

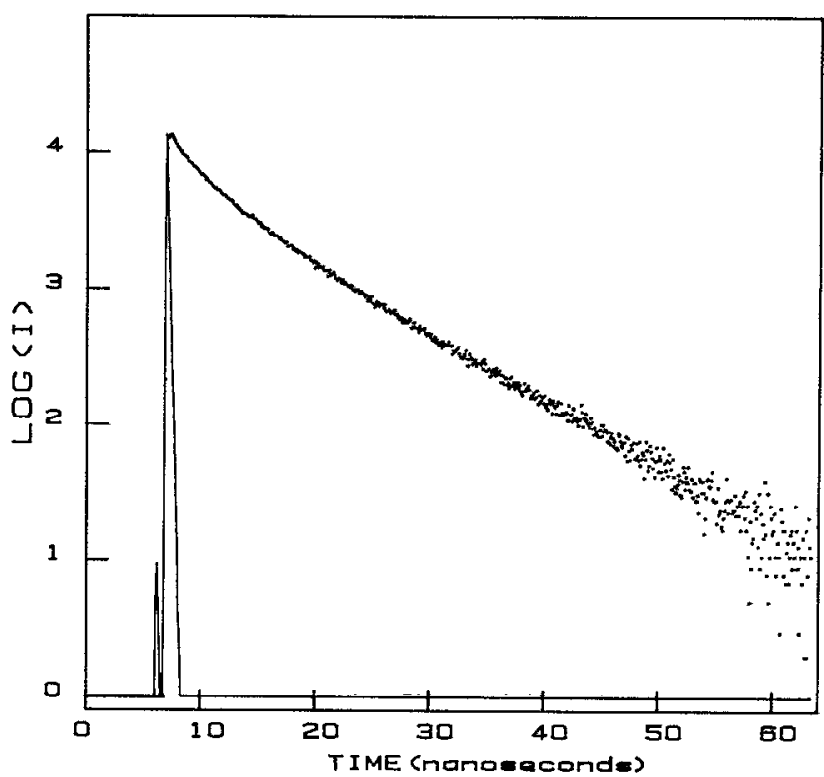

FIG. 1. The survival probability of 2-methoxynaphthalene, excited at 325 $\mathrm{nm}\left(30770 \mathrm{~cm}^{-1}\right)$ with emission at $340 \mathrm{~nm}\left(20412 \mathrm{~cm}^{-1}\right)$, adsorbed in silicalite which is deoxygenated and under vacuum ( $1 \mathrm{mTorr})$.

$$
\Phi(t)=\exp -\frac{t}{\tau_{0}} \exp \left[-A g_{d}(a t)\right]
$$

where $A$ is a time-dependent constant

$$
A=V_{d} p \rho_{0} \epsilon^{-d} \text {, }
$$

with $V_{d}$ the volume of the interaction space. In the limit of $a t>1$,

$$
g_{d}(a t)=\ln ^{d}(a t) \text {. }
$$

The survival probability for exchange takes the form of an exponential logarithm damped by the isolated donor lifetime $\mathrm{e}^{18,19}$

$$
\Phi(t)=\exp \left(\frac{-t}{\tau_{0}}\right) \exp \left(-A \ln \frac{d^{t}}{\tau_{0}}\right) .
$$

The exchange term can be shown to be approximated at long times by an enhanced power of the form ${ }^{13}$

$$
\Phi(t)=\exp \left(-\frac{t}{\tau_{0}}\right) t^{-A\left[\ln \left(t / \tau_{0}\right)\right] d-1} .
$$

It should be noted that neither Eq. (13) nor Eq. (14) are analytical at $t=0$. This is due in part to the approximation in the integral expression equation (2). However, both equations describe exchange in the long time limit; when $d>1$, Eqs. (13) and (14) describe a relaxation which decays slower than a stretched exponential and faster than an algebraic.

The work presented here will focus on the fluorescence emission from excited naphthalene and 2methoxynaphthalene adsorbed to silicalite. The fluorescence survival probability is found to be nonexponential, as shown for 2-methoxynaphthalene in Fig. 1. Nonexponential decay of the emission from molecules adsorbed to the surface of oxides of $\mathrm{Si}$ and $\mathrm{Al}$ has been observed by other 
investigators. $^{9-11}$ We provide experimental evidence which suggests that under the appropriate conditions, the excited state of the adsorbed molecule undergoes energy transfer to intrinsic defect sites of the silica and this interaction gives rise to the nonexponential survival probability observed. Furthermorc, we will demonstrate that the required spectral overlap exists between the emission from naphthalene and 2-methoxynaphthalene donors and the absorption of silicliate defect acceptors. We will also show that the donor relaxation is well described by the dipolar mechanism, while the exchange mechanism fails to capture the essential features of the decay process. Values for the lifetime $\tau_{0}$ and the prefactor are determined, and $\tau_{0}$ is shown to depend on the interactions of the adsorbed molecules with the surface. Values for $R_{0}$ and the defect density $\rho_{0}$ are also determined based on the spectral overlap and the value for the energy transfer prefactor. The value for $R_{0}$ for both the adsorbed naphthalene and 2-methoxynaphthalene is found to be approximately $10 \AA$ and the defect concentration is approximately $3 \pm 2 \times 10^{20}$ defects $\mathrm{cm}^{-3}$. This is an order of magnitude greater than the maximum defect density found in quartz $\mathrm{z}^{20-23}$ but is on the order of the number of $\mathrm{Al}$ substitutional disorder (1/unit cell) in silicalite and is less than the number of dangling bonds shown to be on the silicalite interior by nuclear magnetic resonance (NMR) and Fourier transformation infrared (FTIR) measurements. ${ }^{24}$

\section{EXPERIMENT}

\section{Sample preparation}

Silicalite used for these studies was Union Carbide S115 with an $\mathrm{Al}_{2} \mathrm{O}_{3}$ impurity content no greater than 0.4 to $1.6 \mathrm{wt}$. \% and a mean particle size of approximately $1 \mu \mathrm{m}$. Silicalite is synthesized by crystallizing a silica-organic template and removing the organic component by chemical or thermal decomposition. ${ }^{25}$ Prior to use, the as received silicalite was heated to $600^{\circ} \mathrm{C}$ under dry air for $20 \mathrm{~h}$ to remove any residual template molecules and to dehydrate the surface.

Naphthalene and 2-methoxynaphthalene were obtained from Aldrich and used without further treatment. These were adsorbed to the silicalite from iso-octane solutions. As it is difficult to desorb solvents from silicalite, we choose iso-octane to avoid solvent contamination in our samples, since it has been demonstrated previously that iso-octane does not adsorb into silicalite pores in appreciable quantities even at elevated temperature. ${ }^{26}$ Adsorption was allowed to take place in sealed air tight vials where the samples were allowed to sit without agitation until equilibrium was reached (approximately $300 \mathrm{~h}$ ). Equilibrium was determined by measuring the uptake of naphthalene as a function of time over a two week period. The supernatant was decanted from the samples and the wet powder was dried under vacuum at $50^{\circ} \mathrm{C}$ for $30 \mathrm{~min}$. The uptake of the organic by the solids was calculated from the difference between the optical absorption of the starting solutions and that of the supernatant. To remove any adsorbate remaining on the silicalite particle surface dried silicalite samples were washed in neat iso-octane ( $5 \mathrm{ml}$ per gram of solid) for

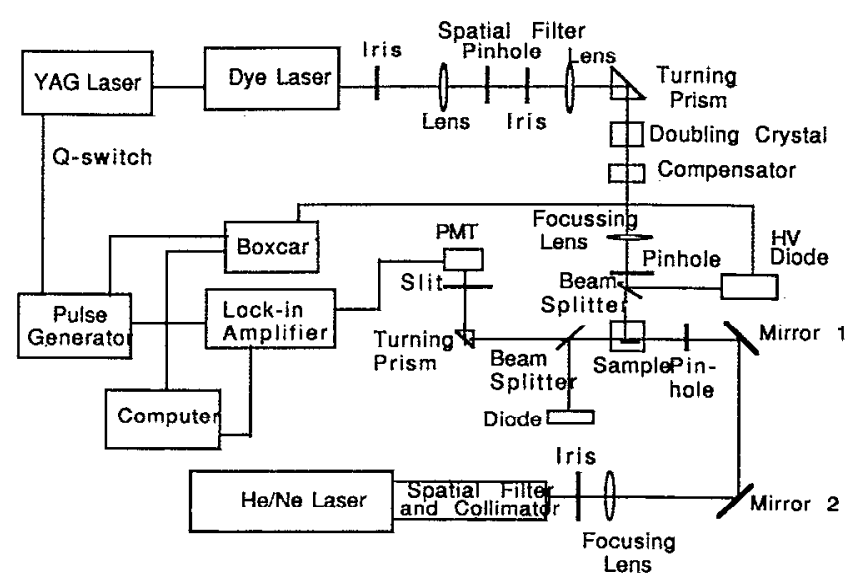

FIG. 2. A schematic diagram of the photothermal deflection apparatus.

$30 \mathrm{~min}$. The wash was decanted and measured for adsorbate content, while the samples were dried for $1 \mathrm{~h}$ as above.

The naphthalene samples used for these studies contained between 3 and $10 \mu \mathrm{M} / \mathrm{g}$ adsorbate and the 2methoxynaphthalene samples contained $54 \mu \mathrm{M} / \mathrm{g}$. The samples were stored in air tight vials at room temperature. The 2-methoxynaphthalene samples were stored in the dark to avoid photodegradation. We found that all samples could be kept for months without any change in their photophysical behavior.

\section{Photothermal deflection measurement}

The absorption spectrum of silicalite defects from 278 $\mathrm{nm}\left(35970 \mathrm{~cm}^{-1}\right)$ to $337 \mathrm{~nm}\left(29674 \mathrm{~cm}^{-1}\right)$ was measured using photothermal deflection spectroscopy. ${ }^{27,28}$ The silicalite was pressed into a $<500 \mu \mathrm{m}$ thick disk and suspended in iso-octane solution at room temperature. As shown in Fig. 2, the $10 \mathrm{~ns}$ output of a $10 \mathrm{~Hz}$ Nd Yag pumped dye laser was frequency doubled and used to excite the defects. The temperature change in the iso-octane due to nonradiative thermal relaxation of the excited defects at the surface of the sample was probed using a HeNe ( $632 \mathrm{~nm}, 15924 \mathrm{~cm}^{-1}$ ) beam focused ( $<50 \mu$ m diameter) $100 \mu \mathrm{m}$ away from the surface of the sample. The deflected beam was then passed over a knife edge $35 \mathrm{~cm}$ from the sample. The a.c. component of the deflection signal was detected using a photomultiplier tube (PMT) and lock-in amplifier referenced to the $Q$ switch of the YAG laser. The signal was collected and averaged for $100 \mathrm{~s}$ and was corrected for the intensity of the excitation source at the sample. The energy density used in these experiments was approximately $7.5 \mathrm{~mJ}$ pulse $\mathrm{cm}^{-1} \mathrm{~cm}^{-2}$. The deflected signal was shown to depend linearly with energy density for values under approximately $10 \mathrm{~mJ}$ pulse $\mathrm{s}^{-1} \mathrm{~cm}^{-2}$. This is important because high energy densities of UV light have been shown to produce defects in quartz through multiphoton processes. $^{21,29,30}$ 


\section{Time-resolved emission measurements}

The decay of the sample luminescence was monitored using time-correlated single photon counting (TCSPC). A Coherent mode-locked argon ion laser synchronously pumping a cavity dumped dye laser provided a $757 \mathrm{kHz}$ train of 5 ps [full width at half-maximum (FWHM)] pulses at $575 \mathrm{~nm}\left(17391 \mathrm{~cm}^{-1}\right)$ which was frequency doubled to $287.5 \mathrm{~nm}\left(34783 \mathrm{~cm}^{-1}\right)$ and used to excite the sample. The energy density of light used for these experiments was $<1 \mathrm{~nJ}$ pulse ${ }^{-1} \mathrm{~cm}^{-2}$. The emission was collected through a $320 \mathrm{~nm}\left(31250 \mathrm{~cm}^{-1}\right)$ cut-off filter and a monochromator set to the desired wavelength. Emissions was detected with a Hamatsu R2023M two stage microchannel plate photomultiplier tube. The signal was collected by an Ortec 50 time-to-amplitude converter (TAC). The anode pulse was amplified and fed to a Tennelec 454 constant fraction discriminator which created a start pulse for the TAC. The stop pulse was provided by the laser pulse to insure a stop pulse for every start pulse. The correlation photon count rate $(<2000 \mathrm{cps})$ from the TAC was transferred to a pulse height analyzer (Tracor TN$1750)$. During the measurements, the samples were under vacuum ( $<1 \mathrm{~m}$ Torr).

The temporal dependence of sample luminescence decay was fit to the model discussed below by a nonlinear least-squares optimization using a Marquadt-Levenberg algorithm. Minimization was done on the $\chi^{2}$ function and the calculated values of the theoretical model were used as weighing factors. Different statistical functions including $\chi^{2}(=1$ and 1.5$)$, the Durbin Watson ( $\left.D W=1.7-2\right)$, the skewness factor $(\mathrm{SK}=0)$, the kurtosis factor $(\mathrm{Kur}=3)$, the weighted residual, and the autocorrelation of the weighted residual were calculated to determine the uniqueness of the fit to the model equations.

\section{Steady-state emission measurements}

Steady-state emission spectra of the samples were taken using an SLM 4800S emission spectrometer with a mercury/xenon lamp and monochromator as the source. Sample emission was collected at a $90^{\circ}$ angle to the excitation beam through a monochromator and detected by a photomultiplier tube. The emission level was normalized to the intensity of the excitation light using a Rhodamine B quantum counter. The spectra were corrected for the wavelength response of the detection system. For this study, the spectra were obtained at room temperature. Samples were contained in sealed quartz tubes that had been evacuated for $1 \mathrm{~h}$ at $<1 \mathrm{~m}$ Torr as described above. The silicalite samples prepared this way contained some residual oxygen. This affected only the signal intensity and not the spectral information.

\section{RESULTS AND DISCUSSION}

The system investigated is comprised of organic probe molecules, naphthalene, and 2-methoxynaphthalene adsorbed to silicalite, a crystalline silica zeolite. Silicalite has a density of $1.76 \mathrm{~g} / \mathrm{cm}^{3}$ and a refractive index of $1.39 .{ }^{25} \mathrm{It}$

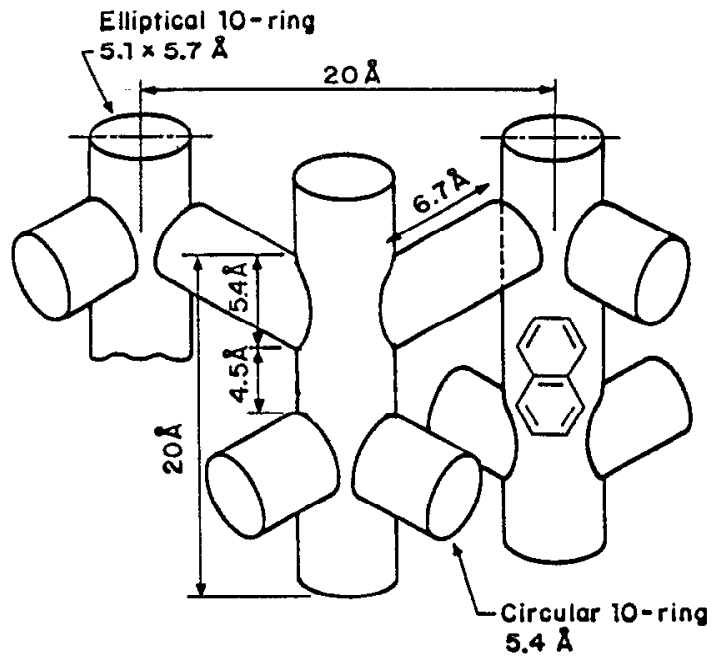

FIG. 3. A schematic representation of the pore network of silicalite with naphthalene in the $b$ channel.

is similar to the ZSM-5 zeolites, although it has a much lower $\mathrm{Al}$ content $\left(\mathrm{SiO}_{2} / \mathrm{Al}_{2} \mathrm{So}_{4}>200\right)$. Five fivemembered rings and one six-membered ring make up the basic repeat unit of silicalite. The repeat units are arranged to form a regular network of channels $5.5-5.8 \AA$ in diameter. There are two types of channels-straight $B$ channels and sinusoidal $A$ and $C$ channels-which intersect the $B$ channels every $10 \AA$. The unit cell of silicalite is defined by $96 \mathrm{Si}\left(\mathrm{O}_{4}\right)_{1 / 2}$ tetrahedra of our $10 \AA$ segments of each the $A, B$, and $C$ channels, This is illustrated in Fig. 3 which is a schematic of the silicalite pore network with an adsorbed naphthalene. ${ }^{26}$

While silicalite has a highly connected pore network, simulations have indicated that aromatic adsorbates lie primarily in the $B$ channels and are sterically hindered from going into the $A$ and $C$ channels. ${ }^{31}$ A ChemX representation of 2-methoxynaphthalene adsorbed in the $B$ channel of silicalite is presented in Fig. 4 and shows that the silicalite pore width is only slightly larger than the molecular width. Adsorption studies indicate that the adsorption isotherm for naphthalene saturates around $100 \mu \mathrm{M} / \mathrm{g}$ or 0.6 molecules per unit cell, which is significantly less than the theoretical maximum of four per unit cell. The samples used for these studies had significantly less than the maximum adsorbate levels. At loadings higher than $100 \mu \mathrm{M} / \mathrm{g}$, we found that the dynamic characteristics changed and this change is attributed to aggregation of adsorbates on the crystallite surfaces.

Recent work investigating the silicalite in the absence of adsorbates has shown that there are defects throughout the silicalite just as there are in all silica substance. ${ }^{32}$ The defects may be in the form of nonbridging oxygen atoms $\mathrm{Si}-\mathrm{O}$, peroxide radical and bridges $\mathrm{Si}-\mathrm{O}_{2}$ and $\mathrm{Si}-\mathrm{O}-\mathrm{O}-\mathrm{Si}$ and oxygen vacancies $=\mathrm{Si}-\mathrm{Si}=$, and the $E^{\prime}$ center. ${ }^{33,34}$ Many studies have been conducted on these defects to show that they absorb subbandgap light..$^{30,33-36}$ They also emit light in the range $300-650 \mathrm{~nm}\left(33333-15385 \mathrm{~cm}^{-1}\right)$. Assignments of the different defect types to distinct absorp- 


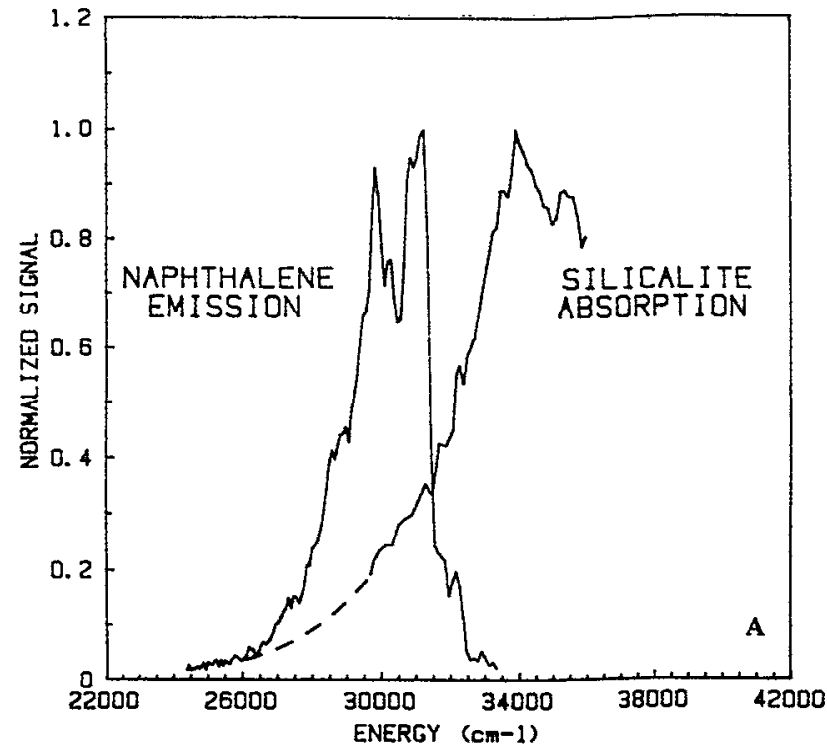

FIG. 4. The optical absorption spectrum of the silicalite defects measured by photothermal deflection spectroscopy. The steady state fluorescence emission spectrum of naphthalene adsorbed in silicalite (excitation 34843 $\mathrm{cm}^{-1}$ and $287 \mathrm{~nm}$ ).

tion and emission bands are not completely understood and are the subject of continuing research.

We have conducted a series of experiments to measure the absorption of silicalite using photothermal deflection spectroscopy. ${ }^{32}$ We find overlapping peaks at approximately 293 and $283 \mathrm{~nm}\left(34130\right.$ and $35336 \mathrm{~cm}^{-1}$ ) (see Fig. 5) that we assign to a peroxide radical or peroxide bridge defect. Such defects have recently been identified in quartz using electron-spin resonance (ESR) techniques ${ }^{37}$ and theoretical calculations show that their absorption en-

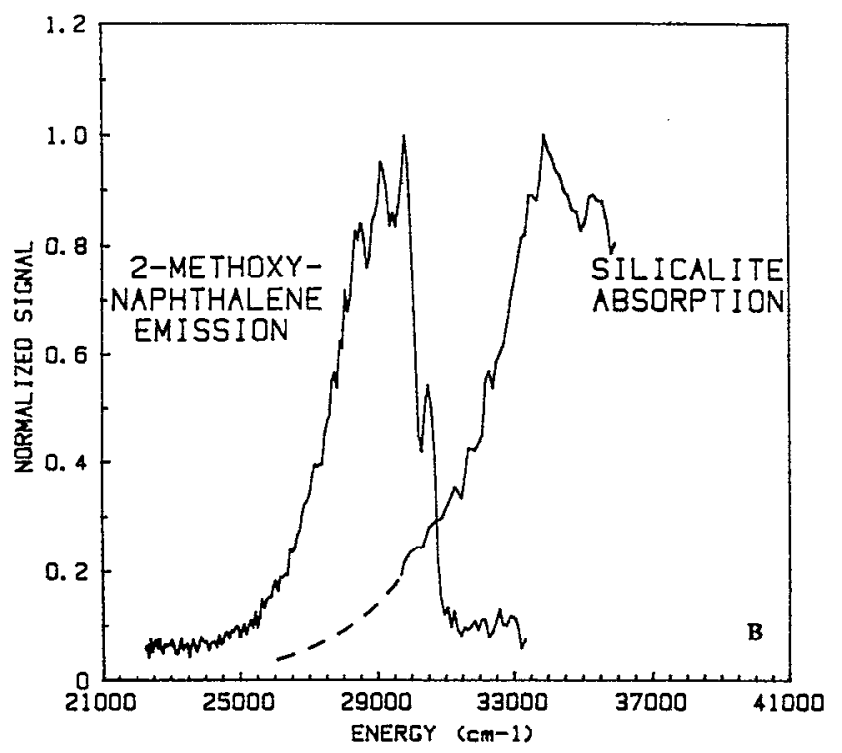

FIG. 5. The optical absorption spectrum of the silicalite defects measured by photothermal deflection spectroscopy. The steady state fluorescence emission spectrum of 2-methoxynaphthalene adsorbed in silicalite (excitation $34843 \mathrm{~cm}^{-1}$ and $287 \mathrm{~nm}$ ). ergy is in the range we have probed. ${ }^{38}$ These defects are known to be caused by the reaction of molecular oxygen at oxygen vacancy defect sites. ${ }^{37,39}$ Our heating of the silicate under oxygen gas may have enhanced the production of these defects in the system.

Defects in zeolites may also be located at impurity sites such as $\mathrm{Al}, \mathrm{Ge}$, and $\mathrm{Fe}$. In silicalite, the primary impurity is $\mathrm{Al}$ and the $\mathrm{SiO}_{2} / \mathrm{Al}_{2} \mathrm{O}_{3}$ ratio of the silicalite used in these studies indicates that the $\mathrm{Al}$ density is as high as one atom per unit cell, or that as many as $1 \%$ of all $\mathrm{Si}$ atoms are replaced by $\mathrm{Al}$. In the crystal, the $\mathrm{Al}$ impurities are associated with $\mathrm{Na}^{+}$or $\mathrm{H}^{+}$counterions creating a large dipole moment of approximately $10 \mathrm{D}$ at the defect which is similar in size to the dipole moment created in the excited aromatic molecule. Some studies have suggested that absorption and emission properties may be attributed to these sitcs. $^{33,34}$

The spectral overlaps in the region $408-287 \mathrm{~nm}$ (24 $500-36000 \mathrm{~cm}^{-1}$ ) between the absorption of silicalite and the emission from naphthalene and 2-methoxynaphthalene are shown in Fig. 5. For the silicalite absorption to the red of $337 \mathrm{~nm}\left(29674 \mathrm{~cm}^{-1}\right)$, we have extrapolated from the data by fitting the absorption edge to an exponential function in keeping with the Urbach model. ${ }^{40,41}$ The overlap between the silicalite and the adsorbate emission is significant, confirming that the dipole-dipole coupling model may be appropriate to describe the interaction. In order to evaluate $R_{0}$ with these data, we estimate the molar extinction coefficient $\epsilon$ for the silicalite. Value for both the absolute absorption $\alpha\left(\mathrm{cm}^{-1}\right)$, and the concentration of defects in the silicalite $\rho_{A}$ are obtained. Photothermal deflection (PTD) spectroscopy allows us to estimate the value for $\alpha$, but we have found that it is difficult to obtain a suitable reference standard to calibrate the instrument, as scattering effects vary widely for different materials and are difficult to account for quantitatively. Estimations for both $\alpha$ and $\rho_{A}$ are determined below by using both the spectral overlap data and the survival probability results.

The survival probabilities of the excited naphthalene and 2-methoxynaphthalene adsorbed to silicalite are shown in Fig. 6. In both cases, the relaxation is clearly nonexponential and is fit to Eq. (6), where $d=3$ and $S=6$. By setting $d=3$, we ignore the excluded volume suggested by the pore geometry as will be addressed below. Figure 7 shows the same data as in Fig. 6 fit to the model for an exchange interaction [Eq. (13)]. Clearly, unlike the dipole-dipole coupling model, the exchange model does not describe the basic temporal features of the relaxation process.

In the case of naphthalene, fitting the fluorescence relaxation to Eq. (6) yields $A_{0}=0.80 \pm 0.06$ and $\tau_{0}=68.0$ $\pm 2.3 \mathrm{~ns}$, while in the case of 2-methoxynaphthalene, $A_{0}$ $=0.98 \pm 0.02$ and $\tau_{0}=14.3 \pm 0.1$. These values are independent of the adsorbate loading within the range 1$100 \mu \mathrm{M} / \mathrm{g}$. For very low loadings, the silicalite emission contributes a large fraction to the total emission, and at high loading, adsorbate aggregation alters the dynamics. We should also note that the silicalite absorption overlaps with the naphthalene absorption, but has a significantly 

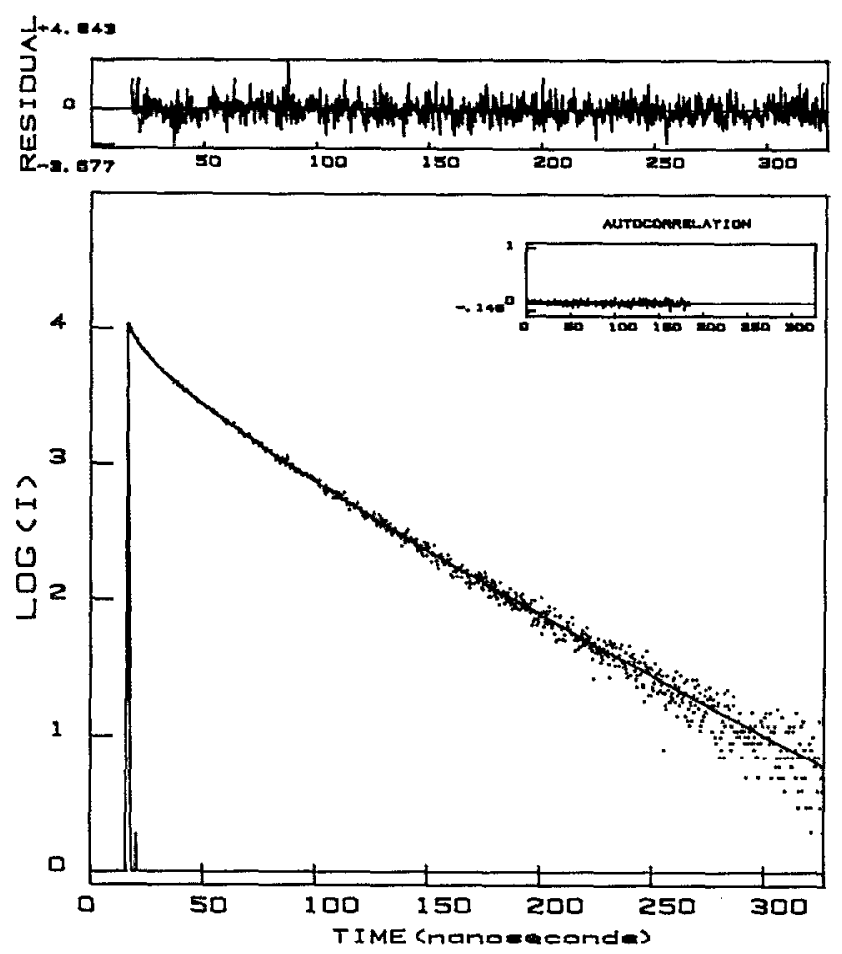

FIG. 6. Survival probability of naphthalene measured at $340 \mathrm{~nm}$ (29 410 $\left.\mathrm{cm}^{-1}\right)$ with excitation at $287 \mathrm{~nm}\left(34843 \mathrm{~cm}^{-1}\right)$. The survival probability is fit (solid line) using two adjustable parameters $\tau_{0}$ and $A_{0}$ to the dipoledipole energy transfer model [Eq. (6)] where $d=3$ and $S=6$. The fit to the data is shown by the residuals and their autocorrelation and $\chi^{2}=1.19$, $\mathrm{DW}=1.88, \mathrm{SK}=0.22$, and $\mathrm{Kur}=3.33$. The narrow peak near the origin is the instrument response function to the excitation pulse.

smaller cross section ( $\epsilon_{\max } \leqslant 100$ for silicalite compared with $\epsilon_{\max }=6000$ for naphthalene), so that even with the population of silica defects greater than that of the adsorbates, most of the light is absorbed by the adsorbates. We have demonstrated that for the samples used in these studies, the silicalite contributed $<1 \%$ of the total emission.

Based on Eqs. (5) and (6), the values for $\rho_{0}$ and $R_{0}$ in the system may be calculated using the values measured for $A_{0}$ and the overlap integral. Again, the value of the absolute absorption must be estimated in order to carry out the analysis. We used a value of $100 \mathrm{~cm}^{-1}$ for $\alpha_{\max }$ and quantum yield values of 0.2 for naphthalene and 0.38 for 2methoxynaphthalene. We find that $\rho_{0}$ is $3 \pm 2 \times 10^{20} \mathrm{~cm}^{-3}$, $R_{0}=10 \AA$ for naphthalene donors, and $R_{0}=10.5 \AA$ for 2-methoxynaphthalene donors. Given these results, the value for $\epsilon_{\max }$ is calculated to be $300 \mathrm{~cm}^{-1} \mathrm{M}^{-1}$. This value is consistent with that observed by Devine who has studied multiphoton effects in quartz. ${ }^{21} \mathrm{He}$ has found that for a defect density of $3-6 \times 10^{15} \mathrm{~cm}^{-3}, \alpha_{\max }(258 \mathrm{~nm})=0.002$, which is equivalent to $\epsilon_{\max }$ of $200-400 \mathrm{~cm}^{-1} \mathrm{M}^{-1}$. Naturally, this comparison does not verify our results as the absolute values of $\alpha$ and $\rho_{0}$ in silicalite and quartz differ by five orders of magnitude from those found by Devine.

The value of $\rho_{A}$ determined $\left(2 \times 10^{20} \mathrm{~cm}^{-3}\right)$ is equivalent to 1 defect/unit cell or a defect on $1 \%$ of all Si atoms ( $0.25 \%$ of all $\mathrm{Si}-\mathrm{O}$ bonds). Defect density values reported for quartz vary from $10^{14}-10^{20}$ depending on the sample
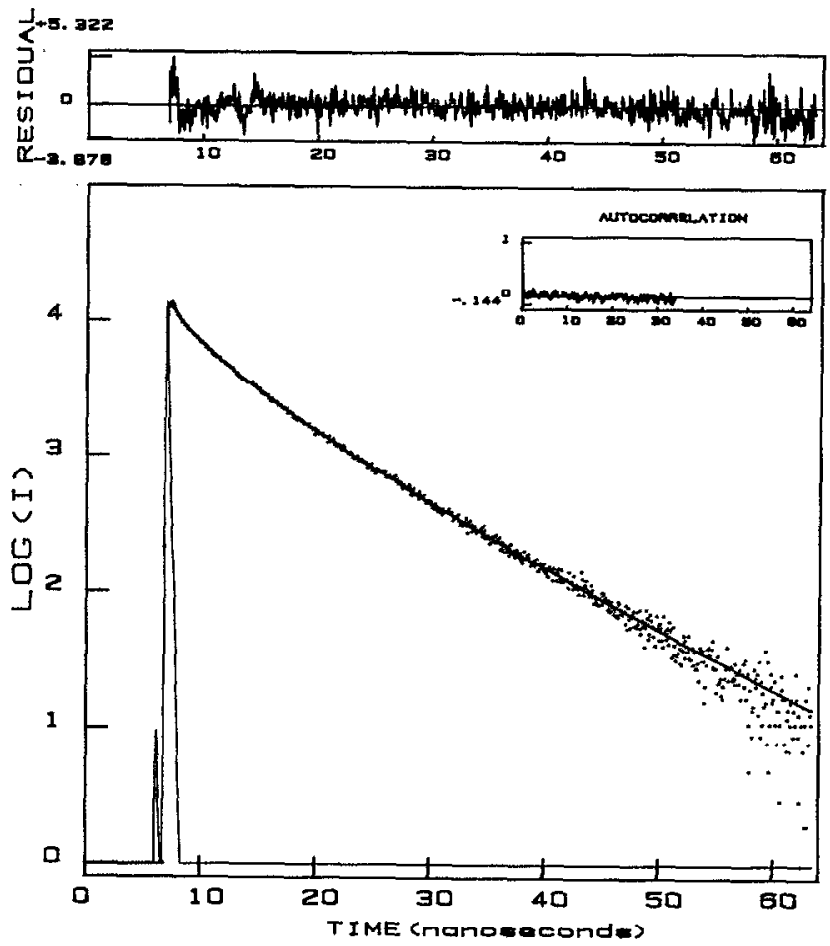

FIG. 7. Survival probability of 2-methoxynaphthalene measured at 340 $\mathrm{nm}\left(29410 \mathrm{~cm}^{-1}\right)$ with excitation at $325 \mathrm{~nm}\left(30770 \mathrm{~cm}^{-1}\right)$. The survival probability is fit (solid line) using two adjustable parameters $\tau_{0}$ and $A_{0}$ to the dipole-dipole energy transfer model [Eq. (6)] where $d=3$ and $S=6$. The fit to the data is shown be the residuals and their autocorrelation and $\chi^{2}=1.60, \mathrm{DW}=1.70, \mathrm{SK}=0.07$, and $\mathrm{Ku}=3.62$. The narrow peak near the origin is the instrument response function to the excitation pulse.

and the technique used. ${ }^{20-23,33}$ Woolery et al. have recently performed NMR and FTIR studies on silicalite and ZSM-5 samples with varying amounts of $\mathrm{Al}^{24}$ They find that the number of dangling bonds on the silicate interior increases significantly with decreasing $\mathrm{Al}$ content. For extremely low Al content $\left(\mathrm{SiO}_{2} / \mathrm{Al}_{2} \mathrm{O}_{3}=26000\right)$, they found that as many as $7 \%$ of the internal $\mathrm{Si}$ atoms had dangling bonds.

For a defect density of $2 \times 10^{20} \mathrm{~cm}^{-3}$, the average distance between defects is approximately $9.3 \AA$, or on the order of one channel spacing. The size of the defects can be estimated to be on the order of $\mathrm{Si}-\mathrm{O}$ bond length $(2.5 \AA)$ for the lattice defects and slightly larger for the $\mathrm{Al} \mathrm{impu-}$ rity defects. On the average, the adsorbate molecules interact with defects across one or more channel. Temporally, the system behaves as donors and acceptors embedded in a uniform three-dimensional lattice. The adsorbates probe the defect density and the energy transfer may be used to characterize the defect distribution in the silicalite.

For dipole-dipole energy transfer, we may calculate the maximum time of the energy transfer process $t_{\max }$, which is determined by the radiative damping process $\tau_{0}$. The maximum distance probed by the energy transfer $R_{\max }$ may be defined as $R_{\max }=\frac{3}{2} \kappa^{2}\left(1 / \tau_{0}\right) R_{0}^{S}{ }^{1,14,16}$ We can show that $R_{\max } \sim 1.45 R_{0}$, which is calculated to be $15 \AA$ for the interaction of naphthalene and 2-methoxynaphthalene with the defects. This means that the excited donor will 

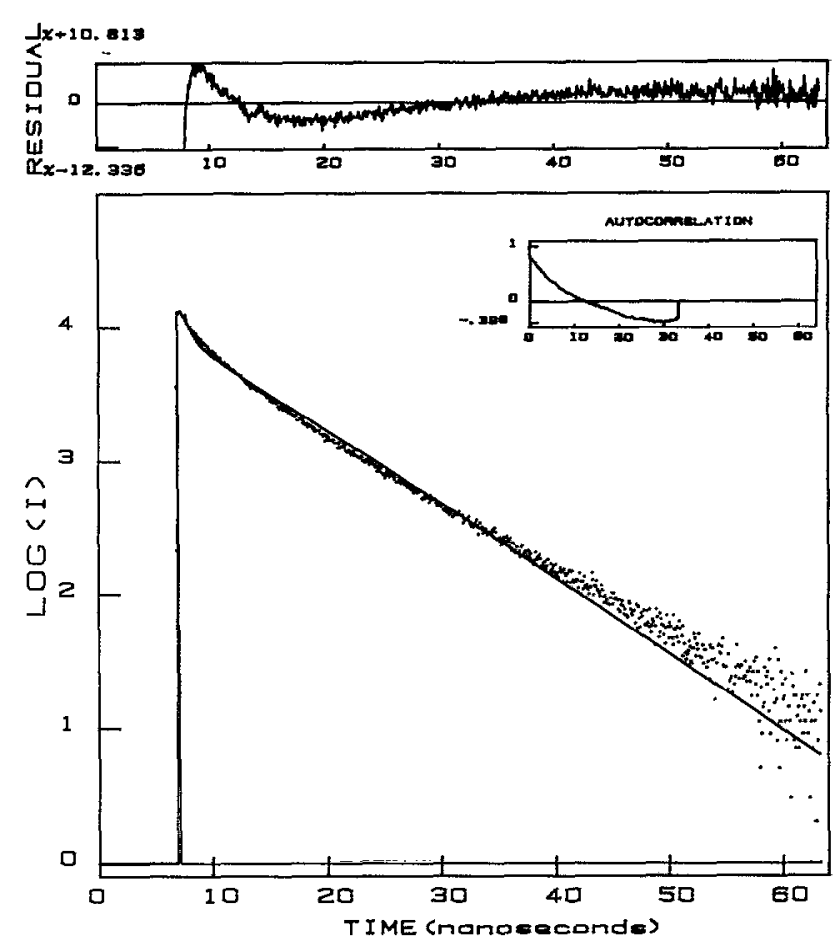

FIG. 8. Survival probability of 2-methoxynaphthalene is the same as in Fig. 7, but fit to the exchange energy transfer model [Eq. (13)] using two adjustable parameters $\tau_{0}$ and $A$, where $d=3$. The goodness of the fit to the data is given by $\chi^{2}=11.71, \mathrm{DW}=0.31, \mathrm{SK}=0.03$, and $\mathrm{Kur}=3.23$.

interact primarily with defects between two channels. The form of the distribution of the acceptor defects will depend on the adsorption site, whether the molecule is in the $B$ channel or an intersection. This will determine the arrangement of $\mathrm{SiO}_{2}$ units and of the excluded volume or probe space around the probe.

For porous media, dipolar energy transfer processes can exhibit a crossover in dimensionality. This crossover is associated with a characteristic time $t_{\text {cross }}$, which in this system is related to a relaxation behavior crossing over from $d<3$ to $d=3$, where excluded volume effects are less important. $t_{\text {cross }}$ may be approximated by $\left(2 R_{p}\right.$ $\left.R_{0}\right)^{6} \tau_{0}, 1,14,16$ where $R_{p}$ is the pore radius which is approximately $2.8 \AA$. Based on the values we determine for $R_{0}$ and $\tau_{0}, t_{\text {cross }} \sim 2 \mathrm{~ns}$ for naphthalene and $0.33 \mathrm{~ns}$ for 2methoxynaphthalene, which confirms that the model with $d=3$ will describe the survival probability.

Finally, the lifetimes determined for the adsorbates using the dipole-dipole coupling model indicate that the adsorption may also affect the inherent fluorescence lifetime of the naphthalene probes. For comparison, we have measured the fluorescence lifetimes of naphthalene and 2methoxynaphthalene in solution. The value obtained for naphthalene in nonpolar iso-octane solution is $104.0 \pm 0.5$ $\mathrm{ns}$, in acrotic acetonitrile it is $93.4 \pm 0.3 \mathrm{~ns}$, and in ethanol it is $93.9 \pm 0.2 \mathrm{~ns}$. The lifetimes are very sensitive to dissolve oxygen, and these values represent the best results obtained by removing oxygen by a freeze-pump-thaw method. They may be compared to the value of $31.1 \pm 1.0$ ns obtained in air-equilibrated ethanol. The lifetime of 2methoxynaphthalene in iso-octane is $14.3 \pm 0.1 \mathrm{~ns}$, while that in acetonitrile is $13.1 \pm 0.1 \mathrm{~ns}$. Clearly, the naphthalene lifetime is shorter for the adsorbed molecule ( 68.0 $\pm 2.3 \mathrm{~ns}$ ) than for that in solution, while the 2methoxynaphthalene lifetime $(14.3 \pm 0.1 \mathrm{~ns})$ is unaffected by adsorption. The steady state emission spectrum for the adsorbed naphthalene is similar to that measured in acetonitrile, indicating that the adsorption process does not affect the emission energy.

\section{CONCLUSIONS}

We have demonstrated that lattice defect sites may interact through a dipolar energy transfer mechanism with excited organic molecules adsorbed in a porous silica (silicalite). We have shown that the silicalite defect absorption overlaps the adsorbate emission and that the survival probability of excited adsorbate molecules may be fit by the dipole-dipole coupling model. The critical radius $R_{0}$ for the interaction between naphthalene donors and the defect acceptors is found to be approximately $10 \AA$ and that for 2-methoxynaphthalene donors is approximately $10.5 \AA$. Although $R_{0}$ is small, suggesting that an exchange mechanism may be significant, the survival probability of the excited donor is not described by an exchange mode, and we may conclude that the dipolar interaction dominates the energy transfer in this system. From the parameters measured, we estimate the defect density to be on the order of $3 \pm 2 \times 10^{20} \mathrm{~cm}^{-3}$.

\section{ACKNOWLEDGMENTS}

The authors would like to thank Professor J. Klafter for many helpful discussions. A. R. L. would like to thank the National Institute of Health for a N.R.S.A. fellowship and Exxon Research and Engineering for generous support of this work.

${ }^{1}$ P. Levitz, J. M. Drake, and J. Klafter, J. Chem. Phys. 89, 5224 (1988).

${ }^{2}$ J. M. Drake, P. Levitz, J. Klafter, N. J. Turro, K. S. Nitsche, and K. F. Cassidy, Phys. Rev. Lett. 61, 865 (1988).

${ }^{3}$ N. J. Turro, I. R. Gould, M. B. Zimmt, and C. C. Cheng, Chem. Phys. Lett. 119, 484 (1985).

${ }^{4}$ C. Yang, M. A. El-sayed, and S. L. Suib, J. Phys. Chem. 91, 4440 (1987).

${ }^{5}$ U. Even, K. Rademann, J. Jortner, N. Manor, and R. Riesfeld, Phys. Rev. Lett. 52, 2164 (1984).

${ }^{6}$ R. Kopelman, Science 241, 1620 (1988).

${ }^{7}$ B. I. Greene and R. R. Millard, Phys. Rev. Lett. 55, 1331 (1985).

${ }^{8}$ O. Pekan, M. A. Winnik, and M. D. Croucher, Phys. Rev. Lett. 61, 641 (1988).

${ }^{9}$ P. K. Bauer, P. de Mayo, L. V. Natarajan, and W. R. Ware, Can. J. Chem. 62, 1279 (1984).

${ }^{10}$ X. Liu, K. K. Iu, and J. K. Thomas, J. Phys. Chem. 93, 4120 (1989).

${ }^{11}$ R. Krasnasky, K. Koike, and J. K. Thomas, J. Phys. Chem. 94, 4521 (1990).

${ }^{12}$ T. Basche and C. Brauchle, J. Phys. Chem. 92, 5069 (1988).

${ }^{13}$ A Blumen, J. Klafter, and G. Kumofen, in Optical Spectroscopy of Glasses, edited by I. Zsehokke (Reidel, New York, 1986).

${ }^{14}$ J. M. Drake, J. Klafter, and P. Levitz, Science 251, 1574 (1991).

${ }^{15}$ T. Forster, Discuss. Faraday Soc. 27, 7 (1959).

${ }^{16}$ P. Levitz, G. Ehret, S. K. Sinha, and J. M. Drake, J. Phys. Chem. 95, 6151 (1991).

${ }^{17}$ M. F. Shlesinger, Annu. Rev. Phys. Chem. 39, 269 (1988).

${ }^{18}$ A. Blumen, J. Chem. Phys. 72, 2632 (1980). 
${ }^{19} \mathrm{M}$. Inokuti and F. Hirayama, J. Chem. Phys. 43, 1978 (1965).

${ }^{2}$ R. C. Hughes, Phys. Rev. B 15, 2012 (1977).

${ }^{21}$ R. A. B. Devine, Phys. Rev. Lett. 62, 340 (1989).

${ }^{22}$ A. J. Moulson and J. P. Roberts, Trans. Brit. Ceram. Soc. 59, 388 (1960).

${ }^{23}$ D. W. Compton and G. W. Arnold, Discuss. Faraday Soc. 31, 130 (1961).

${ }^{24}$ G. I. Woolery, L. B. Alemany, R. M. Dessau, and A. W. Chester, Zeolites 6, 14 (1984).

${ }^{25}$ E. M. Flanigen, J. M. Bennett, R. W. Grose, J. P. Cohen, R. L. Ratton, R. M. Kirchner, and J. V. Smith, Nature 271, 512 (1978).

${ }^{26}$ D. T. Hayhurst and A. R. Prauer, Zeolites 8, 27 (1988).

${ }^{27}$ W. B. Jackson, N. M. Amer, A. C. Boccara, and D. Fournier, Appl. Opt. 20, 1333 (1981).

${ }^{28}$ A. C. Tam, Rev. Mod. Phys. 58, 381 (1986).

${ }^{29}$ T. E. Tsai, D. L. Griscom, and E. J. Friebele, Phys. Rev. Lett. 61, 444 (1988).
${ }^{30}$ J. H. Stathis and M. A. Kastner, Phys. Rev. B 29, 7079 (1984).

${ }^{31}$ R. Reischman, L. D. Schmitt, and D. H. Olson, J. Phys. Chem. 92, 5165 (1988).

${ }^{32}$ A. R. Leheny, N. J. Turro, and J. M. Drake (to be published).

${ }^{33}$ D. L. Griscom, Proceedings of the 32nd Annual Frequency Control Symposium (Electronic Industrial Association, Washington, D.C., 1979).

${ }^{34}$ D. L. Griscom, J. Non-Cryst. Solids 73, 51 (1985).

${ }^{35}$ M. Guzzi, M. Martini, M. Mattaini, F. Pio, and G. Spinolo, Phys. Rev. B 35, 9407 (1987).

${ }^{36}$ R. Tohmon, H. Mizuno, Y. Ohki, K. Sasagane, K. Nagasawa, and Y. Hama, Phys. Rev. B 39, 1337 (1989).

${ }^{37}$ T. E. Tsai and D. L. Griscom, Phys. Rev. Lett. 67, 2517 (1991).

${ }^{38}$ E. P. O'Reilly and J. Robertson, Phys. Rev. B 27, 3780 (1983).

${ }^{39} \mathrm{R}$. L. Pfeffer, in The Physics and Chemistry of $\mathrm{SiO}_{2}$ and the $\mathrm{SiO}_{2}$ Interface (Plenum, New York, 1988).

${ }^{40}$ C. Bosio and W. Czaja, Philos, Mag. B 63, 7 (1991).

${ }^{41}$ A. D. Steward and P. I. Jones, Philos. Mag. B 57, 431 (1988). 